\title{
The next big LEAP2 understanding ghrelin function
}

\author{
Zane B. Andrews
}

Monash Biomedicine Discovery Institute, Metabolism, Diabetes and Obesity Program, Monash University, Clayton, Victoria, Australia.

\begin{abstract}
Chrelin is a key signal driving energy seeking and storage in order to reverse energy deficit. In line with this view, the metabolic status of an organism predicts sensitivity to ghrelin, with fasting increasing and obesity decreasing ghrelin sensitivity. However, the mechanism responsible for controlling this sensitivity is unknown. In this issue of the $J C I$, Mani and colleagues show that plasma levels of plasma liver-enriched antimicrobial peptide-2 (LEAP2), a recently identified hormone that antagonizes the ghrelin receptor, are inversely correlated with those of plasma acyl-ghrelin under conditions of both energy deficit and energy surplus in mice and humans. Their results show that a fall in plasma LEAP2 during energy deficit facilitates the actions of acyl-ghrelin, whereas increased LEAP2 in obesity suppresses the actions of acyl-ghrelin. This important discovery helps reshape our understanding of ghrelin function and may provide a new approach to aiding weight maintenance after diet-induced weight loss.
\end{abstract}

that ghrelin actions are heightened during energy deficit and attenuated in the setting of energy surplus, as in obesity. However, the key question in the field prevails: what is the physiological mechanism or mechanisms responsible for controlling this metabolic sensitivity?

\section{Identifying a modulator of ghrelin sensitivity}

A potential answer to this important question was reported in 2018, when the Kaplan lab used an unbiased RNAsequencing approach and discovered that liver-enriched antimicrobial peptide-2 (LEAP2) mRNA was elevated in the stomach remnant and reduced in the duodenum after vertical sleeve gastrectomy (23). Subsequent characterization of LEAP2 revealed that it was a potent and selective antagonist of GHSR. In vivo studies demonstrated that LEAP2 blocked ghrelin-induced food intake and that virally mediated overexpression of LEAP2 impaired blood glucose maintenance during severe CR, similarly to what is seen in genetic models of ghrelin or GOAT deletion/ablation (4, 13). Moreover, neutralizing antibodies to LEAP2 boosted ghrelin-induced growth hormone release, presumably by increasing endogenous acyl-ghrelin function. Thus, Kaplan and colleagues had serendipitously discovered a potential mechanism underlying the effect of metabolic state on ghrelin sensitivity. In this issue, Mani and colleagues took the next step to elucidate the role of LEAP2 in linking ghrelin's action to metabolic state (24). The authors tested the hypotheses that LEAP2 was a metabolic hormone regulated by body mass, feeding, and blood glucose and that it works in concert with acyl-ghrelin to regulate the activity of GHSR in different metabolic states.

\section{LEAP2 is regulated by} metabolic state

The authors first sought to firmly validate an ELISA method to measure plasma LEAP2. Next, they performed a number 
of analyses to understand the relationship between plasma acyl-ghrelin and LEAP2. They demonstrated that, in mice with diet-induced obesity (DIO), LEAP2 was elevated and positively correlated with fat mass, whereas DIO decreased plasma ghrelin, leading to an increased LEAP2/ acyl-ghrelin ratio (24). Thus, since LEAP2 prevents ghrelin-induced food intake (23), high-plasma LEAP2 during obesity may underlie the ghrelin resistance observed in DIO mice (17-19). Intriguingly, dietinduced weight loss in previously obese mice lowered plasma LEAP2 and the LEAP2/acyl-ghrelin ratio (24), which was consistent with previous studies showing that diet-induced weight loss reverses ghrelin resistance (19). Conversely, fasting, which increases ghrelin sensitivity (25), significantly reduced plasma LEAP2 and increased plasma ghrelin, such that the LEAP2/acyl-ghrelin ratio was significantly reduced.

An increase in blood glucose, as a signal of energy availability, suppresses ghrelin secretion and reduces plasma acylghrelin (26), but the role for LEAP2 in this context was unknown. With this is mind, Mani et al. showed that acute oral glucose gavage increased plasma LEAP2 and decreased acyl-ghrelin within one hour in fasted mice (24), consistent with previous studies showing that glucose pretreatment blocks ghrelin-induced food intake (27). Plasma LEAP2 was positively correlated with blood glucose, whereas acyl-ghrelin was negatively correlated with blood glucose. Collectively, these studies in mice show that LEAP2 is regulated by metabolic state, in opposition to acyl-ghrelin, with higher concentrations in obese states and lower concentrations in fasted states. Using electrophysiological recordings of NPY neurons, Mani et al. demonstrated that LEAP2 acts as a powerful GHSR antagonist, blocking ghrelin-induced activation of NPY neurons and hyperpolarizing NPY neurons by disabling GHSR constitutive activity (24). This work clearly demonstrates that elevated LEAP2 is a key mechanism responsible for ghrelin resistance in obesity. Intriguingly, diet-induced weight loss restores ghrelin sensitivity, which promotes rebound weight gain in a ghrelindependent manner (19), suggesting that LEAP2 after weight loss may help to prevent rebound weight gain.

\section{From mouse to human}

A strength of this manuscript is that Mani and colleagues demonstrated that their studies in mice also held true in humans, suggesting LEAP2 might play important roles in human conditions of metabolic dysfunction. Indeed, obesity in humans was associated with higher fasted LEAP2, lower fasted acyl-ghrelin, and thus a higher LEAP2/acyl-ghrelin ratio. LEAP2 also positively correlated with several clinical parameters of metabolic dysfunction, including BMI, body fat percentage, blood glucose, serum triglycerides, visceral adipose tissue volume, and liver lipid content (24), although there is no evidence to suggest a causal role for plasma LEAP2 in these processes. These results suggest that LEAP2 is a metabolic signal of energy surplus during a state of chronic obesity, but intriguingly, greater postprandial increases in LEAP2 were observed in individuals with higher BMI. This observation suggests that acute energy sensing in the obese state is functionally linked to LEAP2 secretion, an important area for future research. Finally, two types of bariatric surgery, Roux-en-Y gastric bypass (RYGB) and vertical sleeve gastrectomy (VSG), also affected plasma LEAP2. RYGB reduced plasma LEAP2 in the postprandial state 3 months after surgery and in the fasted state 2 years after surgery, whereas VSG lowered postprandial plasma LEAP2 compared with baseline (24).

\section{Future directions}

Like most seminal studies, the work from Mani et al. provides more questions than answers, as it moves a field into a new direction. For example, what are the mechanisms regulating LEAP2 gene expression and secretion from the liver? Can this be modulated by environmental factors, such as macronutrient composition of the diet? Are there actions of LEAP2 that are independent of GHSR antagonism? Is desacyl-ghrelin regulated by LEAP2? Does genetic deletion of LEAP2 heighten ghrelin sensitivity and affect body weight during $\mathrm{CR}$ or DIO? Future studies are required to address these important questions. Nevertheless, this important contribution demonstrates that LEAP2 is a metabolic hormone of energy surplus that regulates plasma ghrelin and ghrelin's action on NPY neurons. Thus, the fall in LEAP2 during negative energy balance removes GHSR antagonism, facilitating the action of acyl-ghrelin to increase food intake, growth hormone secretion and prevent severe hypoglycemia. Conversely, in the setting of obesity, the increase in LEAP2 limits acyl-ghrelin's actions on food intake and blood glucose. More broadly, these studies help us realize that maintenance of energy homeostasis through metabolic feedback is far more complicated than previously appreciated, involving diverse humoral signatures of metabolic state. With this is mind, it's not surprising that early studies looking to pursue ghrelin as an antiobesity therapy produced ambiguous and conflicting results.

Address correspondence to: Zane B. Andrews, Monash University, Rm F215, Building 13F, 26 Innovation Walk, Clayton, Victoria 3800 Australia. Phone: 61.3.9905.8165, 61416.929.263. Email: zane.andrews@monash.edu.

1. Kojima M, Hosoda H, Date Y, Nakazato M, Matsuo H, Kangawa K. Ghrelin is a growthhormone-releasing acylated peptide from stomach. Nature. 1999;402(6762):656-660.

2. Mani BK, Zigman JM. Ghrelin as a survival hormone. Trends Endocrinol Metab. 2017;28(12):843-854.

3. Nakazato $\mathrm{M}$, et al. A role for ghrelin in the central regulation of feeding. Nature. 2001;409(6817):194-198.

4. McFarlane MR, Brown MS, Goldstein JL, Zhao TJ. Induced ablation of ghrelin cells in adult mice does not decrease food intake, body weight, or response to high-fat diet. Cell Metab. 2014;20(1):54-60.

5. Sun Y, Asnicar M, Saha PK, Chan L, Smith RG. Ablation of ghrelin improves the diabetic but not obese phenotype of ob/ob mice. Cell Metab. 2006;3(5):379-386.

6. Sun Y, Butte NF, Garcia JM, Smith RG. Characterization of adult ghrelin and ghrelin receptor knockout mice under positive and negative energy balance. Endocrinology. 2008;149(2):843-850.

7. Wortley KE, et al. Genetic deletion of ghrelin does not decrease food intake but influences metabolic fuel preference. Proc Natl Acad Sci U S A. 2004;101(21):8227-8232.

8. Wortley KE, et al. Absence of ghrelin protects against early-onset obesity. J Clin Invest. 2005;115(12):3573-3578.

9. Zigman JM, et al. Mice lacking ghrelin receptors resist the development of diet-induced obesity. J Clin Invest. 2005;115(12):3564-3572.

10. Zorrilla EP, et al. Vaccination against weight gain. Proc Natl Acad Sci U S A. 2006;103(35):13226-13231.

11. Zigman JM, Bouret SG, Andrews ZB. Obesity impairs the action of the neuroendocrine ghrelin system. Trends Endocrinol Metab. 
2016;27(1):54-63.

12. Briggs DI, Andrews ZB. Metabolic status regulates ghrelin function on energy homeostasis. Neuroendocrinology. 2011;93(1):48-57.

13. Zhao TJ, et al. Ghrelin O-acyltransferase (GOAT) is essential for growth hormone-mediated survival of calorie-restricted mice. Proc Natl Acad Sci U S A. 2010;107(16):7467-7472.

14. Bayliss JA, et al. Ghrelin-AMPK signaling mediates the neuroprotective effects of calorie restriction in Parkinson's disease. J Neurosci. 2016;36(10):3049-3063.

15. Hornsby AK, et al. Short-term calorie restriction enhances adult hippocampal neurogenesis and remote fear memory in a Ghsr-dependent manner. Psychoneuroendocrinology. 2016;63:198-207.

16. Lutter $\mathrm{M}$, et al. The orexigenic hormone ghrelin defends against depressive symptoms of chronic stress. Nat Neurosci. 2008;11(7):752-753.

17. Briggs DI, Enriori PJ, Lemus MB, Cowley MA, Andrews ZB. Diet-induced obesity causes ghrelin resistance in arcuate NPY/AgRP neurons. Endocrinology. 2010;151(10):4745-4755.

18. Briggs DI, et al. Evidence that diet-induced hyperleptinemia, but not hypothalamic gliosis, causes ghrelin resistance in NPY/AgRP neurons of male mice. Endocrinology. 2014;155(7):2411-2422.

19. Briggs DI, Lockie SH, Wu Q, Lemus MB, Stark $\mathrm{R}$, Andrews ZB. Calorie-restricted weight loss reverses high-fat diet-induced ghrelin resistance, which contributes to rebound weight gain in a ghrelin-dependent manner. Endocrinology 2013;154(2):709-717.

20. Lockie SH, Dinan T, Lawrence AJ, Spencer SJ, Andrews ZB. Diet-induced obesity causes ghrelin resistance in reward processing tasks. Psychoneuroendocrinology. 2015;62:114-120.

21. Finger BC, Dinan TG, Cryan JF. Diet-induced obesity blunts the behavioural effects of ghrelin: studies in a mouse-progressive ratio task. Psychopharmacology (Berl). 2012;220(1):173-181.

22. Naznin F, et al. Diet-induced obesity causes peripheral and central ghrelin resistance by promoting inflammation. JEndocrinol. 2015;226(1):81-92

23. Ge XC, et al. LEAP2 is an endogenous antagonist of the ghrelin receptor. Cell Metabolism. 2018;27(2):461-469.e6.

24. Mani BK, et al. LEAP2 changes with body mass and food intake in humans and mice. JClin Invest. 2019;129(9):3909-3923.

25. Hewson AK, Dickson SL. Systemic administration of ghrelin induces Fos and Egr-1 proteins in the hypothalamic arcuate nucleus of fasted and fed rats. J Neuroendocrinol. 2000;12(11):1047-1049.

26. Sakata I, et al. Glucose-mediated control of ghrelin release from primary cultures of gastric mucosal cells. Am J Physiol Endocrinol Metab. 2012;302(10):E1300-E1310.

27. Lockie SH, et al. Glucose availability predicts the feeding response to ghrelin in male mice, an effect dependent on AMPK in AgRP neurons. Endocrinology. 2018;159(11):3605-3614. 